\title{
Efficient CIEDE2000-based Color Similarity Decision for Computer Vision
}

\author{
Américo Pereira, Pedro Carvalho, Member, IEEE, Gil Coelho, and Luís Côrte-Real, Member, IEEE
}

\begin{abstract}
Color and color differences are critical aspects in many image processing and computer vision applications. A paradigmatic example is object segmentation, where color distances can greatly influence the performance of algorithms. Metrics for color difference have been proposed in the literature, including the definition of standards such as the CIEDE2000, which quantifies the change in visual perception of two given colors. This standard has been recommended for industrial computer vision applications, but the benefits of its application have been impaired by the complexity of the formula. This paper proposes a new strategy that improves the usability of the CIEDE2000 metric when a maximum acceptable distance can be imposed. We argue that, for applications where a maximum value, above which colors are considered to be different, can be established, then it is possible to reduce the amount of calculations of the metric, by preemptively analysing color features. This methodology encompasses the benefits of the metric while overcoming its computational limitations, thus broadening the range of applications of CIEDE2000 in both computer vision algorithms and computational resource requirements.
\end{abstract}

Index Terms-Computer Vision, Segmentation, CIEDE2000, Color Similarity.

\section{INTRODUCTION}

C OLOR similarity decision can be seen as the process of calculating the difference between colors and provide a (binary) decision on their similarity. This process has a huge importance in many computer vision (CV) applications such as object segmentation, detection or appearance matching, where it is often an initial step in the processing chains. In an industrial context, color similarity decision is being applied in diverse topics like mineral identification [1], dental ceramics [2], wine color analysis [3] or corrosion detection [4].

The CIEDE2000 [5] formulation is an international standard since 2014, with reference ISO CIE 11664-6:2014 (CIE S 014-6/E:2013), and has been receiving increased interest. It has been recommended for industry applications, showing high potential in discriminating between colors, especially those with higher similarity, since it is closely related to the subjective visual difference $(\Delta V)$ perceived by humans [6]. Nevertheless, its wider usage has been impaired due to the high computation cost, which prevents its application in

Copyright (C)2019 IEEE. Personal use of this material is permitted. However, permission to use this material for any other purposes must be obtained from the IEEE by sending an email to pubs-permissions@ieee.org.

All the authors are with the Centre for Telecommunications and Multimedia at INESC TEC - Institute for Systems and Computer Engineering, Technology and Science, Porto, Portugal; (email: americo.j.pereira@inesctec.pt, pedro.carvalho@inesctec.pt, agcoelho@inesctec.pt, lreal@inesctec.pt).

A.Pereira and L. Côrte-Real are also with Faculty of Engineering, University of Porto, Porto, Portugal.

P. Carvalho is also with the School of Engineering of the Polytechnic Institute of Porto, Porto, Portugal. many scenarios, particularly those with hardware or real time constraints [7]. Moreover, applications that need to perform multiple $\Delta E_{00}$ calculations, e.g. video processing, will be seriously affected with high computation times. Even though the technological evolution has enabled higher computation capabilities, there is a large number of scenarios that remain infeasible due to the high quantities of visual information that must be processed on restricted hardware.

For many applications that require the calculation of the CIEDE2000 metric to obtain color similarities, only values bellow a given boundary are important as they represent colors considered perceptually similar. All other pairs whose distance is above the boundary can be disregarded, as they are considered different. In this paper we approach this scenario and propose novel strategies that enable a reduction of the amount of times the CIEDE2000 metric needs to be calculated on these types of applications, while maintaining the same exact results obtained using only the CIEDE2000. To achieve this, we propose the usage of simple properties that, when coupled with a color distance limit, can be used to reduce the number of CIEDE2000 calculations.

The remainder of this article is structured as follows. In Section II a brief description of the CIEDE2000 color similarity metric is presented, followed by an analysis of applications that use this metric. Section III formulates and validates the underlying hypothesis of this paper through a mathematical analysis. A further analysis of the properties introduced in Section III is explored in Section IV, coupled with the introduction of a new paradigm for an efficient color similarity decision. The assessment of the proposals put forward in this article are detailed on Section V. Finally, the conclusions and a discussion of the concepts detailed through the article are presented in Section VI.

\section{CIEDE2000 AND ITS USAGE}

The CIEDE2000 [5] metric was designed to characterize human perceptual color differences. The main formula is presented in Eq. 1, where the parameters $(\Delta L, \Delta C, \Delta H)$ correspond to the differences between lightness, chromaticity and hue, respectively. The parameters $\left(S_{L}, S_{C}, S_{H}\right)$ represent weighting functions and $\left(K_{L}, K_{C}, K_{H}\right)$ represent the correction terms. Finally, the $R_{T}$ parameter is the rotation function that accounts for the interference between the chroma and hue differences in the blue portion of the space. For a detailed explanation of the formula and its parameters the reader is referred to [8], with additional implementation and testing material in [9]. 


$$
\Delta E_{00}=\sqrt{\left(\frac{\Delta L}{K_{L} S_{L}}\right)^{2}+\left(\frac{\Delta C}{K_{C} S_{C}}\right)^{2}+\left(\frac{\Delta H}{K_{H} S_{H}}\right)^{2}+R_{T}\left(\frac{\Delta C}{K_{C} S_{C}}\right)\left(\frac{\Delta H}{K_{H} S_{H}}\right)},
$$

The International Commission on Illumination (CIE) also proposed other metrics [10], such as CIE76 $\left(\Delta E_{a b}^{*}\right)$ [11], CIE94 $\left(\Delta E_{94}^{*}\right)$ [12], DeltaE CMC $\left(\Delta E_{C M C}^{*}\right)$ [13] and DeltaE2000_PF [14]. However the CIEDE2000 metric $\left(\Delta E_{00}\right)$ stands out from the others as it is used in many areas and industries.

Color comparison is a basic process present in many image processing algorithms and application. For instance, identifying and measuring color differences is very important in dental applications, where it has been widely studied in the literature. Such applications include color acceptability and perceptibility [15], [16], color accuracy and precision [17], [18] and description of coverage error of dental shade guides [19], [20]. A dentistry study comparing visual and instrumental shade matching was performed in [21], using three CIE color similarity metrics, namely, the older CIELAB $\left(\Delta_{a b}^{*}\right)$, the CIEDE2000(1:1:1), where $K_{L}=1, K_{C}=1$ and $K_{H}=1$ and the $\operatorname{CIEDE} 2000(2: 1: 1)$, where $K_{L}=2, K_{C}=1$ and $K_{H}=1$. This study recommended CIEDE2000(2:1:1) stating it provides a closer representation of the visual perception. Other proposals in the literature stated that $K_{L}=2.3$ obtained better results [22]. However, recent studies [7], [23] still use the value of 1.0 for the CIEDE2000 parametric factors. Based on this, we will use this value in our analysis presented on Section III. In [2], $\Delta E_{00}$ was also compared with other metrics to study the perceptual and acceptability thresholds for dental ceramics, and it was concluded that $\Delta E_{00}$ was able to obtain a good fit for the color difference thresholds.

Even though many different technologies have been applied to mineral identification, including X-ray diffraction and electron microscopy, manual observation is still one of the most employed methods, due to its low cost and speed, but above all because of its reliability [1]. To enable an automated and more efficient mineral identification, a new method was proposed in [7] with the goal of imitating human made mineral identification by applying image processing techniques. It employed different optical properties, including color, interference color and opacity. For the color-based features, the CIELAB color space and the CIEDE2000 color difference metric were applied. Very high results were obtained for the tested materials (above 90\%) and it was reported that the success of the method was largely due to the use of CIELAB color space and the CIEDE2000 metric. However, the authors indicated the metric's high computational time as a drawback.

A saturation test image design method was proposed in [24] that uses the CIEDE2000 metric to generate test images with different saturation but equal lightness and hue. The results show that subjective visual perception is consistent with the saturation test images. Another study comparing the $\operatorname{CIELAB}\left(\Delta_{a b}^{*}\right)$ and CIEDE2000 $\left(\Delta E_{00}\right)$ color differences is presented in [23] for the purpose of evaluating the two formulas and identify the one that better reflects the perceptual color differences, and whether color perception is affected by gender. Results show that CIEDE2000 performs closer to the human color perception.

A color image quality assessment metric was described in [25], where $\Delta E_{00}$ was combined with the printing industry standards for visual verification in order to create a subjective assessment metric. The metric was denominated $Q$ and was stated as a precise objective score conformed to subjective perception (OSCSP).

The super resolution (SR) algorithm presented in [26] employed $\Delta E_{00}$ to generate color difference maps that in turn were used to compare SR results to ground truth. A hue linear color space transformation that maximizes the degree of perceptual uniformity was presented in [27] using the CIELAB color space as basis and then applying two transformations: the first maps the initial color space into a Euclidean space by an optimization methodology that minimizes the disagreement between stored CIEDE2000 color differences and corresponding euclidean distances; the second maps the first resulting color space into a hue linear space.

The edge detection methodology presented in [28] combined edge maps from different sources in order to upscale depth information with limited spatial resolution. Some alternatives were shown and the one that provided the best results was comprised of a combination of edge maps for intensity and color difference. The latter was obtained using CIEDE2000 color difference, in order to obtain a perceptual color edge detection that follows the characteristics of the human visual system. Although this solution provided the best results, it had the cost of a high computational complexity. For color similarity decisions using $\Delta E_{00}$, values equal or bellow 3 are typically applied; for values above 10, two colors are considered to be very different.

\section{PRoperty Definition AND CIEDE2000 RElations}

The important benefits of the $\Delta E_{00}$ metric are often impaired by its complexity. By analysing the $\Delta E_{00}$ formula (presented in Section II and described in detail in [8]), the high complexity of several of the terms is noticeable, resulting in a formula with a large computational cost. Hence, the use of this technique in many application scenarios is often infeasible, particularly when the computational resources are more limited. Moreover, applications that need to perform multiple $\Delta E_{00}$ calculations, e.g. video processing, will be seriously affected with high computation times.

Often, applications that use the $\Delta E_{00}$ metric define a decision boundary and only consider as similar colors bellow such value. Examples of this can be seen in dentistry [21] or mineral extraction [7]. On the other hand, colors above the decision boundary are considered different. We argue that, 
if two colors are distant enough, so that their difference can be identified without requiring the full calculation of the $\Delta E_{00}$ metric, then it is possible to achieve performance gains. With this insight we argue that, if such a maximum value for color similarity is given, then it is possible to preemptively perform an analysis of the color pairs and decide whether the $\Delta E_{00}$ calculation is in fact needed or the colors are too dissimilar and no further calculation is required. We propose that: if such a limit for the $\Delta E_{00}$ is given, then it is possible to assess the expected similarity between two colors through simple filtering modules. With this process, an assessment can be made: (1) the input colors are too dissimilar and no further calculation is required; (2) the previous decision cannot be made and the $\Delta E_{00}$ calculation is necessary. With this approach, the number of $\Delta E_{00}$ calculations may be effectively reduced, thus providing a lower processing time for the full process while assuring the correct decision results.

To achieve this, it is necessary to identify properties that are related to the $\Delta E_{00}$ and satisfy the following assumptions:

1- Are discriminative when comparing colors;

2- Are computationally less expensive than the $\Delta E_{00}$ formula;

3- Any decision boundary defined for the $\Delta E_{00}$ can be mapped to a cut-off value for the given filtering property, as illustrated in Fig. 1.

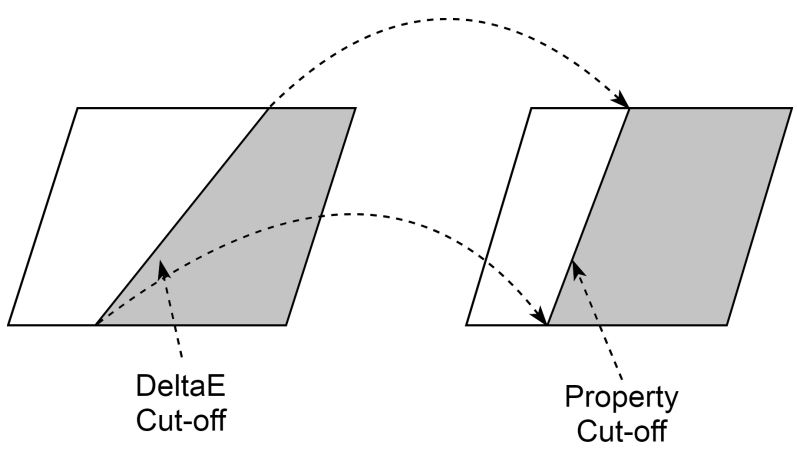

Fig. 1. Mapping of the $\Delta \mathrm{E}_{00}$ cut-off into a corresponding property cut-off The space in grey represents the pixel comparisons in which the $\Delta \mathrm{E}_{00}$ value is above the given cut-off, (i.e., represent different colors).

\section{A. Filtering Properties}

Computer vision applications that work with color images often load them using the RGB color space. This is not agnostic to the fact that displays show images using that color space. Because of that, many algorithms are designed to initially work with this color space. Examples range from mere background subtraction with the traditional MOG algorithm [29] to modern semantic segmentation algorithms such as Mask R-CNN [30], where the common principle is that the input images that are all in the RGB color space. Due to this fact, applications that require the usage of the $\Delta E_{00}$ metric will mostly require the conversion of the loaded images to the CIELAB color space, introducing additional time delays.

With this insight, we formulate the following hypothesis: For a given property $P$ that complies to the aforementioned assumptions, we argue that there is at leat one mapping function such that for a given decision boundary for the $\Delta E_{00}$ metric it produces a corresponding cut-off on the given property. A mathematical formulation of this hypothesis is illustrated in Eq. 2.

$$
\begin{array}{r}
\forall t_{\Delta E} \in \mathbb{R}^{+}, \forall C_{1}, C_{2} \in \mathbb{C}_{R G B}, \\
P\left(C_{1}, C_{2}\right)>f\left(t_{\Delta E}\right) \Longrightarrow \Delta E_{00}\left(C_{1}, C_{2}\right)>t_{\Delta E}
\end{array}
$$

where $t_{\Delta E}$ is the cut-off limit for the $\Delta E_{00}, C_{1}$ and $C_{2}$ are two input colors in the RGB color space represented as $\mathbb{C}_{R G B}$, and $f$ is a mapping function that satisfies assumption 3 and it may include a transformation of the color space.

Following the aforementioned assumptions for a property, we selected two simple properties related to the RGB color space. Namely the angle between RGB vectors and the euclidean distance between them. The selection of these properties is mainly due to their calculation simplicity, but also by their proved discriminative capabilities as demonstrated in [31], [32] and the fact that by using properties in the RGB color space an additional color conversion step is required. Although more complex, an additional property was also selected, namely the lightness dissimilarity of the colors. Unlike the previous two properties, the third one can only be obtained by partially converting the colors to the CIELAB color space. Due to this fact, the third property is expected to be more complex than the remaining properties, but its usage is encouraged by its strong discriminative capability, since the $\mathrm{L}$ component on this color space is closely related with the human perception of lightness. Throughout the remainder of this paper these properties will be referred to as Angle $(\mathcal{A})$, Module $(\mathcal{M})$ and Lightness $(\mathcal{L})$, respectively. Given two colors in RGB, $C_{1}=\left\langle r_{1}, g_{1}, b_{1}\right\rangle$ and $C_{2}=\left\langle r_{2}, g_{2}, b_{2}\right\rangle$, the Angle, Module and Lightness properties are calculated as follows:

$$
\begin{gathered}
\mathcal{A}\left(C_{1}, C_{2}\right)=\arccos \left(\frac{C_{1} \cdot C_{2}}{\left\|C_{1}\right\|\left\|C_{2}\right\|}\right) \\
\mathcal{M}\left(C_{1}, C_{2}\right)=\left\|C_{2}-C_{1}\right\| \\
\mathcal{L}\left(C_{1}, C_{2}\right)=\left|L\left(C_{2}\right)-L\left(C_{1}\right)\right|,
\end{gathered}
$$

where $L$ is a function that computes just the lightness coordinate of the conversion between the RGB color space to the CIELAB [33].

\section{B. Defining a Lower Bound Function}

Given the complexity of the $\Delta E_{00}$ formula, the hypothesis put forward in Eq. 2 can be proven by demonstrating its validity for a lower bound $\left(\Delta E^{\prime}\right)$ function of $\Delta E_{00}\left(\Delta E^{\prime} \leq\right.$ $\left.\Delta E_{00}\right)$ :

$$
P>f\left(t_{\Delta E}\right) \Longrightarrow \Delta E^{\prime}>t_{\Delta E} \Longrightarrow \Delta E_{00}>t_{\Delta E}
$$

By applying variable substitution to the $\Delta E_{00}$ formula (Eq. 1) we can set $a=\frac{\Delta L}{K_{L} S_{L}}, b=\frac{\Delta C}{K_{C} S_{C}}$ and $c=\frac{\Delta H}{K_{H} S_{H}}$, and get the following equation:

$$
\Delta E_{00}=\sqrt{a^{2}+b^{2}+c^{2}+R_{T} b c},
$$


where $R_{T}$ is given by:

$$
R_{T}=-R_{c} \times \sin (2 \Delta \theta),
$$

and is bounded by $-R_{C}$ and $R_{C}$, in which

$$
R_{C}=2 \sqrt{\frac{\bar{C}^{\prime 7}}{\bar{C}^{\prime 7}+25^{7}}}
$$

The $\bar{C}^{\prime}$ variable is the mean chromaticity between the input colors, which is always positive. Thus, we can derive the following upper limit:

$$
\lim _{\bar{C}^{\prime} \rightarrow \infty} R_{C}=2 \sqrt{\frac{\bar{C}^{\prime 7}}{\bar{C}^{\prime 7}+25^{7}}}=2
$$

Hence, $\left|R_{T}\right|<2$. Analysing the possible variations of the term $R_{T} b c$, namely $R_{T} b c \geq 0$ or $R_{T} b c<0$, we get that:

$$
a^{2}+b^{2}+c^{2}-R_{T} b c>a^{2}
$$

Consequently we obtain the following lower bound function $\Delta E^{\prime}$ :

$$
\Delta E^{\prime}=\sqrt{a^{2}}=\sqrt{\left(\frac{\Delta L}{K_{L} S_{L}}\right)^{2}}
$$

\section{Hypothesis Validation}

Given the lower bound function $\Delta E^{\prime}$ (Eq. 12) we can analyse the suggested properties and mathematically verify the validity of our hypothesis. For the purpose of this demonstration, we assume that the mapping function $f$ used in all the proofs is linear, and has the form:

$$
f\left(t_{\Delta E}\right)=m \times t_{\Delta E}+w
$$

We start with the proof for the Lightness property and we wish to prove that:

$$
\mathcal{L}>\left|m t_{\Delta E}+w\right| \Longrightarrow \Delta E^{\prime}>t_{\Delta E}
$$

In this case, we use the absolute value because the difference in lightness can be negative due to the order of the color pairs and we must comply to the commutative property present on the $\Delta E_{00}$ formula. Rewriting Eq. 12, we get that:

$$
\Delta E^{\prime}=\frac{1}{K_{L} S_{L}} \sqrt{\mathcal{L}^{2}}
$$

since $\mathcal{L}^{2}=\Delta L^{2}$. Combining Eqs. 14 and 15 we can get a new minimizing form $\left(\Delta E_{L}\right)$ of $\Delta E_{00}$ :

$$
\begin{array}{r}
\Delta E_{L}=\frac{1}{K_{L} S_{L}} \sqrt{\left(m t_{\Delta E}+w\right)^{2}} \\
\Delta E_{L}=\frac{1}{K_{L} S_{L}} \sqrt{\left(m t_{\Delta E}\right)^{2}+2 m t_{\Delta E} w+w^{2}}
\end{array}
$$

If we select $m$ and $w$ such that:

$$
\left\{\begin{array}{l}
2 m t_{\Delta E} w+w^{2} \geq 0 \\
\frac{m}{K_{L} S_{L}}>1
\end{array}\right.
$$

we get that:

$$
\begin{aligned}
\Delta E_{L} & >t_{\Delta E} \\
\therefore \Delta E_{L}>t_{\Delta E} \Longrightarrow \Delta E & >t_{\Delta E}
\end{aligned}
$$

For the Module and Angle properties, we need to account for color conversion, since $\Delta E_{00}$ uses the LAB color space and these properties are calculated in the RGB color space. This conversion requires an intermediate step converting RGB to the XYZ color space, which in turn requires a white point reference. Due to general acceptance, we used the standard RGB color space and corresponding D65 reference white point through these profs. An important factor in this transformation is that the RGB values must be normalized. From the derivation of the lower bound function for $\Delta E_{00}$ we obtained an expression depending only on the Lightness component of the LAB color space. Thus, we can focus on the conversion for this component, where:

$$
\begin{gathered}
L=116 f_{y}-16 \\
f_{y}= \begin{cases}\sqrt[3]{y_{r}} & \text { if } y_{r}>\epsilon \\
\frac{k y_{r}+16}{116} & \text { otherwise }\end{cases} \\
y_{r}=\frac{Y}{Y_{r}},
\end{gathered}
$$

where $\epsilon=\frac{116}{24389}, k=\frac{24389}{27}$ and $Y_{r}=1$.

$$
Y=\alpha R+\beta G+\gamma B
$$

where $\alpha=\frac{0.2126729}{255}, \beta=\frac{0.7151522}{255}$ and $\gamma=\frac{0.072175}{255}$ are normalizing constants obtained from the standard D65 lighting.

As with the Lightness property, we wish to prove that a function exists such that:

$$
\mathcal{M}>f\left(t_{\Delta E}\right) \Longrightarrow \Delta E^{\prime}>t_{\Delta E}, \forall_{t_{\Delta E} \geq 0} .
$$

Taking Eq. 15, we know that $\Delta L=\left|L_{2}-L_{1}\right|$, where $L$ is defined as:

$$
L\left(y_{r}\right)= \begin{cases}116 \sqrt[3]{y_{r}}-16 & \text { if } y_{r}>\epsilon \\ k y_{r} & \text { otherwise, }\end{cases}
$$

The parameter $y_{r}$ is obtained from Eq. 23, and has a range of values between $[0,1]$. By analysing the derivatives of the Lightness function $L\left(y_{r}\right)$ in the limits of $y_{r}$, we can specify a lower bound function for the Lightness function as:

$$
L^{\prime}\left(y_{r}\right)=\frac{116}{3} y_{r}
$$

This leads to a new $\Delta E_{00}$ lower bound, as:

$$
\Delta E^{\prime \prime}=\sqrt{\left(\frac{\Delta L^{\prime}}{K_{L} S_{L}}\right)^{2}},
$$

where $\Delta L^{\prime}=L_{2}^{\prime}-L_{1}^{\prime}$. Using Eq. 28 for the Module property:

$$
\mathcal{M}>f\left(t_{\Delta E}\right) \Longrightarrow \sqrt{\left(\frac{\Delta L^{\prime}}{K_{L} S_{L}}\right)^{2}}>t_{\Delta E}
$$

By expanding Eq. 28:

$$
\begin{aligned}
& \sqrt{\left(\frac{\Delta L^{\prime}}{K_{L} S_{L}}\right)^{2}}=\sqrt{\left(\frac{\frac{116}{3}\left(y_{r 2}-y_{r 1}\right)}{K_{L} S_{L}}\right)^{2}} \\
& =\left(\frac{116}{3 K_{L} S_{L}}\right) \sqrt{\left(\alpha\left(r_{2}-r_{1}\right)+\beta\left(g_{2}-g_{1}\right)+\gamma\left(b_{2}-b_{1}\right)\right)^{2}},
\end{aligned}
$$


Since $\gamma<\alpha<\beta$ :

$$
\begin{gathered}
\sqrt{\left(\frac{\Delta L^{\prime}}{K_{L} S_{L}}\right)^{2}}>\left(\frac{116 \gamma}{3 K_{L} S_{L}}\right) \sqrt{\mathcal{M}^{2}+Z} \\
Z=2\left(r_{2}-r_{1}\right)\left(g_{2}-g_{1}\right) \\
\quad+2\left(r_{2}-r_{1}\right)\left(b_{2}-b_{1}\right) \\
+2\left(g_{2}-g_{1}\right)\left(b_{2}-b_{1}\right)
\end{gathered}
$$

As with the Lightness, we apply Eq. 29 and get a new lower bound $\Delta E_{M}$ :

$$
\Delta E_{M}=\left(\frac{116 \gamma}{3 K_{L} S_{L}}\right) \sqrt{\left(m t_{\Delta E}\right)^{2}+2 m t_{\Delta E} w+w^{2}+Z}
$$

If we select $m$ and $w$ such that:

$$
\left\{\begin{array}{l}
2 m t_{\Delta E} w+w^{2}+Z \geq 0 \\
\frac{116 \gamma}{3 K_{L} S_{L}} m>1
\end{array}\right.
$$

we get:

$$
\begin{aligned}
\Delta E_{M} & >t_{\Delta E} \\
\therefore \Delta E_{M}>t_{\Delta E} \Longrightarrow \Delta E & >t_{\Delta E}
\end{aligned}
$$

Finally looking into the Angle property, we hypothesise that the following implication holds:

$$
\mathcal{A}>m \times t_{\Delta E}+w \Longrightarrow \sqrt{\left(\frac{\Delta L^{\prime}}{K_{L} S_{L}}\right)^{2}}>t_{\Delta E}
$$

Similarly with the Module, we formulate a new $\Delta E$ lower bound, as in Eq. 31. By applying the cosine rule, we can rewrite the equation as:

$$
\Delta E_{A}=\left(\frac{116 \gamma}{3 K_{L} S_{L}}\right) \sqrt{\left\|C_{1}\right\|^{2}+\left\|C_{2}\right\|^{2}-2\left\|C_{1}\right\|\left\|C_{2}\right\| \cos \theta+Z},
$$

where $\theta$ is the angle between the $C_{1}$ and $C_{2}$ colors, which is the same as property $\mathcal{A}$ and $Z$ is obtained from Eq. 32. Due to the RGB color space, this angle can be at most $\frac{\pi}{2}$.

Given the range of values for angle, we can further simplify the equation by taking an approximation of the cosine in the interval $\left[0, \frac{\pi}{2}\right]$ by using the higher bound function $1-\frac{4}{\pi^{2}} \theta^{2}$, resulting in:

$$
\begin{array}{r}
\Delta E_{A}>\left(\frac{116 \gamma}{3 K_{L} S_{L}}\right) \sqrt{\left\|C_{1}\right\|^{2}+\left\|C_{2}\right\|^{2}-2\left\|C_{1}\right\|\left\|C_{2}\right\|+Q} \\
Q=\frac{8}{\pi^{2}}\left\|C_{1}\right\|\left\|C_{2}\right\| \theta^{2}+Z
\end{array}
$$

Since $\left\|C_{1}\right\|^{2}+\left\|C_{2}\right\|^{2}-2\left\|C_{1}\right\|\left\|C_{2}\right\| \geq 0$, we can further simplify Eq. 39:

$$
\Delta E_{A}>\left(\frac{116 \gamma}{3 K_{L} S_{L}}\right) \sqrt{\frac{8}{\pi^{2}}\left\|C_{1}\right\|\left\|C_{2}\right\| \theta^{2}+Z}
$$

As with the Module:

$$
\Delta E_{A}^{\prime}=\left(\frac{116 \gamma}{3 K_{L} S_{L}}\right) \sqrt{\frac{8}{\pi^{2}}\left\|C_{1}\right\|\left\|C_{2}\right\|(m \times t+w)^{2}+Z}
$$

If we select $m$ and $w$ such that:

$$
\left\{\begin{array}{l}
\frac{16}{\pi^{2}} m t_{\Delta E} w\left\|C_{1}\right\|\left\|C_{2}\right\|+\frac{8}{\pi^{2}} w^{2}\left\|C_{1}\right\|\left\|C_{2}\right\|+Z \geq 0 \\
\frac{232 \sqrt{2} \gamma}{3 K_{L} S_{L} \pi} \sqrt{\left\|C_{1}\right\|\left\|C_{2}\right\|} m>1,
\end{array}\right.
$$

we get that:

$$
\begin{aligned}
\Delta E_{A}^{\prime} & >t_{\Delta E} \\
\therefore \Delta E_{A}^{\prime}>t_{\Delta E} \Longrightarrow \Delta E & >t_{\Delta E}
\end{aligned}
$$

With these mathematical derivations, we show that it is possible to obtain at least one mapping function that relates a $\Delta E_{00}$ cut-off to a bounding limit in the proposed properties. While these mapping functions can be as simple as linear models, lower complexity mapping functions may not be as efficient as more complex models.

\section{A New Paradigm for the EfFicient Application OF CIEDE2000}

In the previous section we verified that the Module, Angle and Lightness are eligible properties to help decide a color dissimilarity without computing the full $\Delta E_{00}$ metric. For demonstration purposes linear mapping functions were used. However, since multiple simplifications were made when deriving these models, the impact that can be achieved on taking a decision may not be optimal. Due to this fact, it is possible to derive data-driven models that can be more efficient for certain applications, which would in turn produce a more notable impact. To achieve this, it is necessary to perform a detailed analysis of the proposed properties and their relation to the $\Delta E_{00}$ formula.

To conduct this analysis, unique colors were uniformly sampled from the images of the Caltech 256 [34] dataset and the $\Delta E_{00}$, Module, Angle and Lightness between each unique color pairs was calculated. This resulted in a dataset with 5 million color comparisons. Figs. 2, 3 and 4 illustrate the distribution of the proposed properties in comparison with the actual $\Delta E_{00}$ value for the color comparisons obtained. In order to observe with more detail the relations between the properties and the $\Delta E_{00}$ values, three different $\Delta E_{00}$ cutoffs were considered, namely, 3,5 and 10 . Color differences above the cut-off are represented in dark grey and in light grey otherwise. Moreover, the corresponding boundaries for Module, Angle and Lightness are represented by a horizontal line. From the plots its possible to notice that, for each property cut-off, there is no light-grey value above the threshold line. Hence, a confident decision on the similarity of the colors, based on the filtering modules, can be made for color pairs with values above the horizontal line.

Moreover, the plots in Figs. 2 through 4 show that the thresholds are proportional to the $\Delta E_{00}$ cut-off values. It can also be noticed that the number of color differences that can be filtered is very significant (amount of dark grey points above the line). Note that $\Delta E_{00}$ values above 7 are considered to represent clear color differences (see Table 1 of [25]).

Given these observations, a validation of their usefulness from an application point of view may be very valuable. To do this, we derived two sets of mapping functions for each property, using their analytical deduction, and checked their 


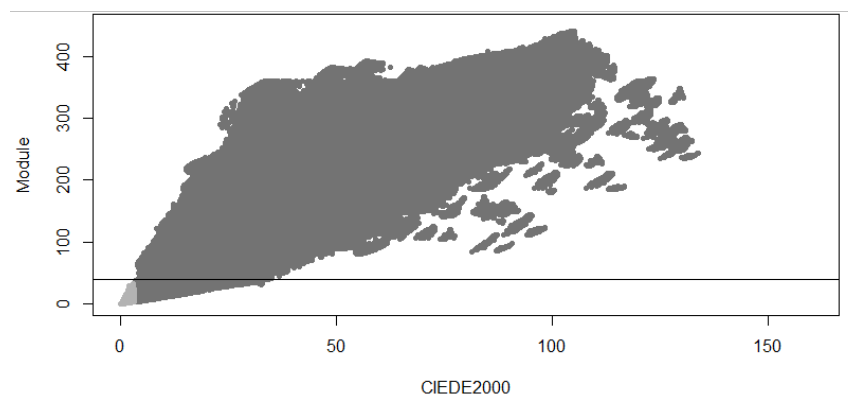

(a) $\Delta E_{00}$ cut-off $=3$

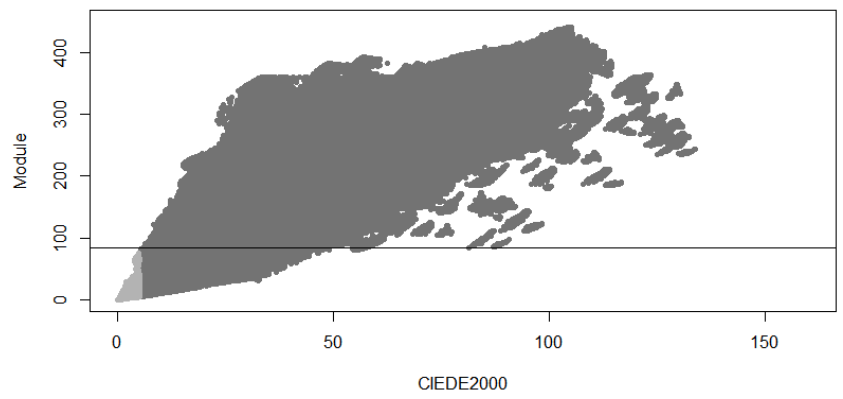

(b) $\Delta E_{00}$ cut-off $=5$

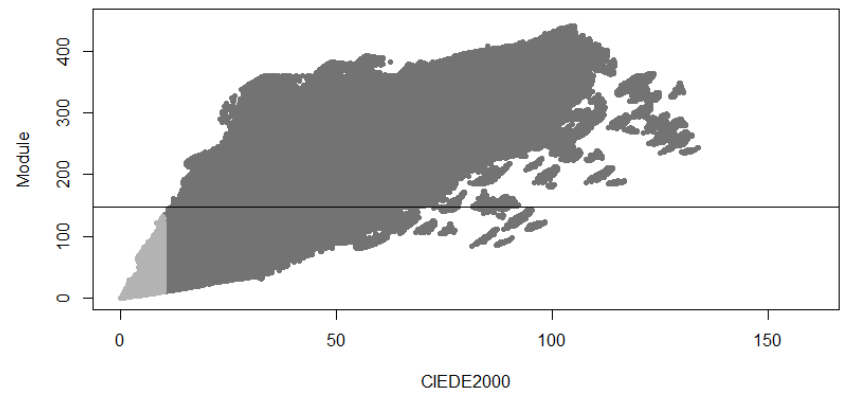

(c) $\Delta E_{00}$ cut-off $=10$

Fig. 2. Relations between results of color differences using $\Delta E_{00}$ and the corresponding value obtained by Module. In dark gray $\Delta E_{00}$ values above the cut-off and in light gray values bellow or equal to the threshold. The horizontal line represents a suggested cut-off for Module.

coverage on our color comparison dataset. We observed that the $\mathcal{L}$ property was able do discriminate between $60 \%$ and $80 \%$ of the color pairs. The $\mathcal{M}$ property was only able to filter $20 \%$ of the colors pairs, and only on the lowest $\Delta E_{00}$ limit. On the other hand, the $\mathcal{A}$ was not able to filter any color. These observations correspond to our expectations, as the $\mathcal{A}$ and $\mathcal{M}$ functions were obtained through the application of several simplifications for the lower bound functions. These discrimination rates were obtained by checking the property and $\Delta E_{00}$ value of each color pair on the dataset and counting the amount of times the property value was above the mapped cut-off. To validate these results we also checked on each of

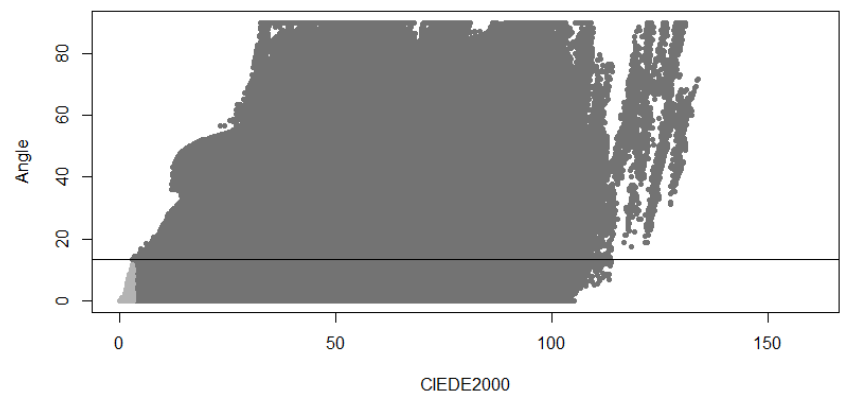

(a) $\Delta E_{00}$ cut-off $=3$

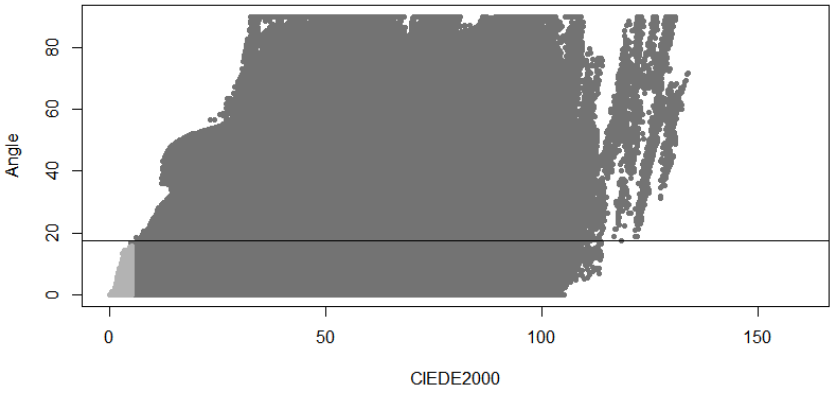

(b) $\Delta E_{00}$ cut-off $=5$

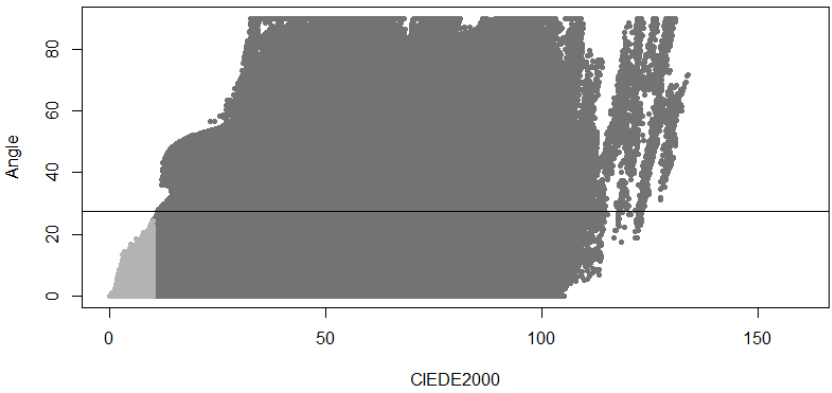

(c) $\Delta E_{00}$ cut-off $=10$

Fig. 3. Relations between results of color differences using $\Delta E_{00}$ and the corresponding value obtained by Angle. In dark gray $\Delta E_{00}$ values above the cut-off and in light gray values bellow or equal to the threshold. The horizontal line represents a suggested cut-off for Angle.

these cases whether the $\Delta E_{00}$ value was also above the corresponding cut-off and; as expected the error rate was null. Due to these observations, we believe that it is possible to derive more efficient mapping functions by employing regressions models from observed data. To achieve this, it is important to better understand the behaviour between the properties and the $\Delta E_{00}$ values, so that correlations can be identified. To perform this assessment, we analysed the color comparisons obtained from the Caltech dataset, and, for each property, we iterated the $\Delta E_{00}$ value between 0 and 30, with an interval of 0.01 units to identify the corresponding maximum value for the property, and plot these relations on Fig. 5. We calculated 


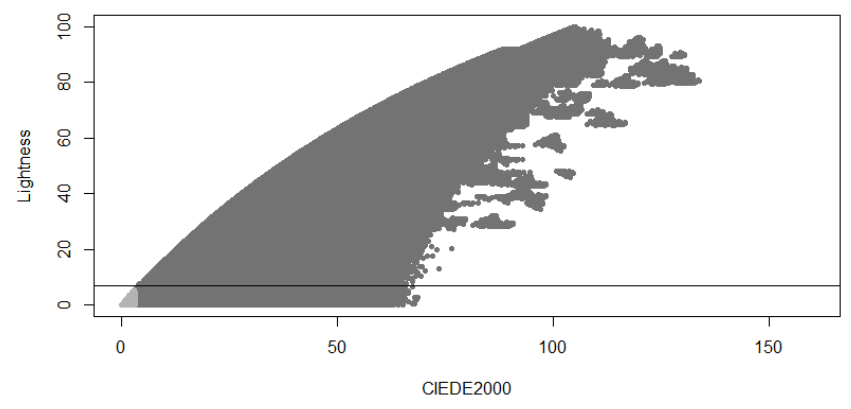

(a) $\Delta E_{00}$ cut-off $=3$

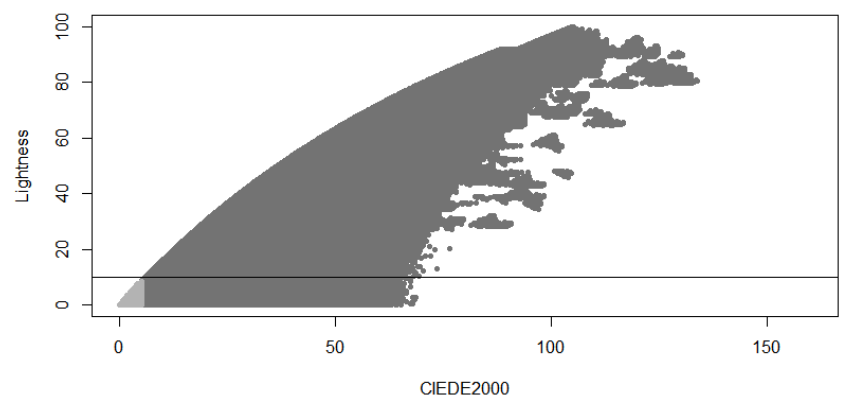

(b) $\Delta E_{00}$ cut-off $=5$

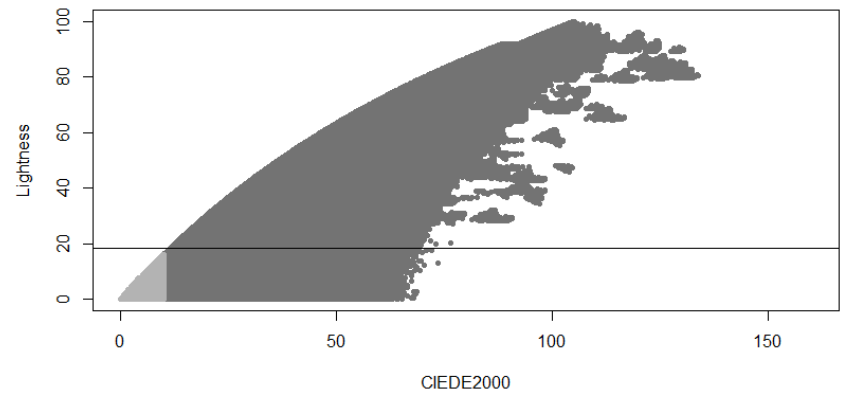

(c) $\Delta E_{00}$ cut-off $=10$

Fig. 4. Relations between results of color differences using $\Delta E_{00}$ and the corresponding value obtained by Lightness. In dark gray $\Delta E_{00}$ values above the cut-off and in light gray values bellow or equal to the threshold. The horizontal line represents a suggested cut-off for Lightness.

the correlation coefficient for each property- $\Delta E_{00}$ pair and obtained the following results: 0.9917425 for Module- $\Delta E_{00}$, 0.9721194 for Angle- $\Delta E_{00}$ and 0.9985428 for Lightness$\Delta E_{00}$. Since the correlation coefficients are very close to 1 and are positive, meaning that they are positively related. These observations show that there are clear relations between the properties and the $\Delta E_{00}$ value, which further suggests that it is possible to train regression models with experimental data in order to obtain better mapping functions. Through the mathematical analysis and observation of the relations between $\Delta E_{00}$ and the $\mathcal{L}$ property, we believe that a linear model for this property can be a good estimator. However, more advanced and complex models may be required for the other properties. While different approaches could be applied, we chose to use a random forest [35] for the $\mathcal{A}$ and $\mathcal{M}$ properties. For all these models, a 10 -fold cross-validation approach was followed and the best RMSE obtained for the models were 0.00675 for the Angle, 0.0333 for the Module and 0.006 for the Lightness.

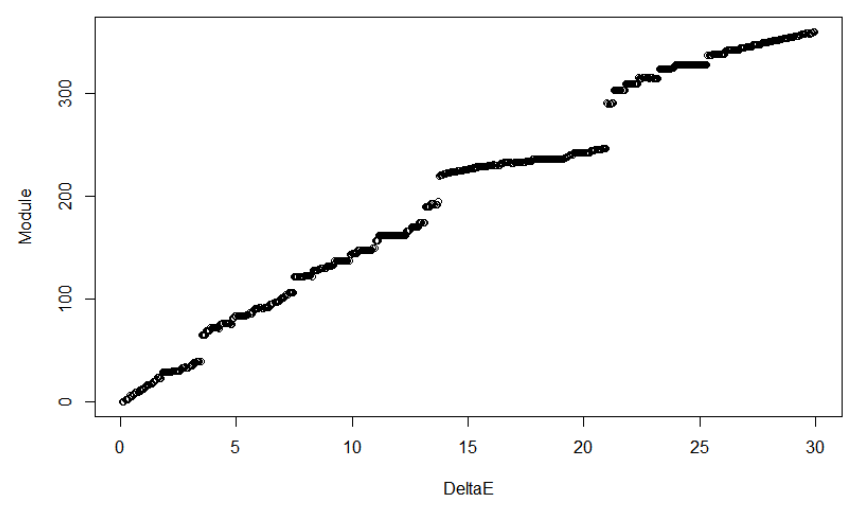

(a) $\Delta E_{00}$ vs. Module cut-offs

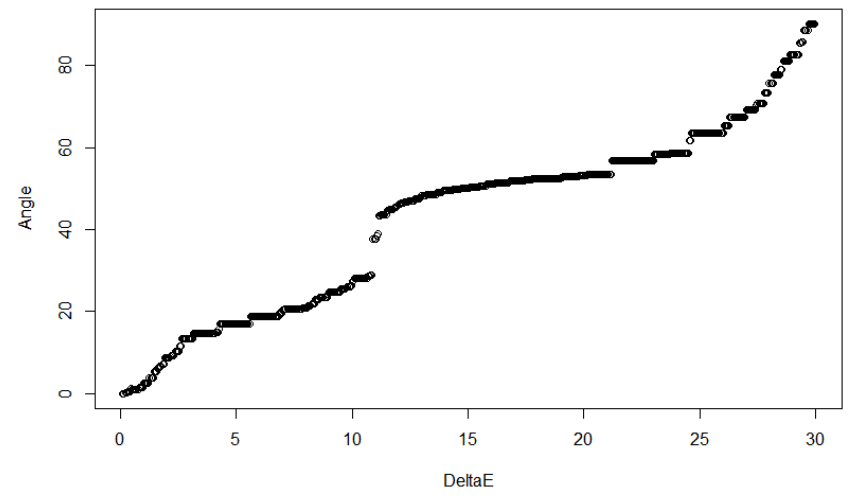

(b) $\Delta E_{00}$ vs. Angle cut-offs

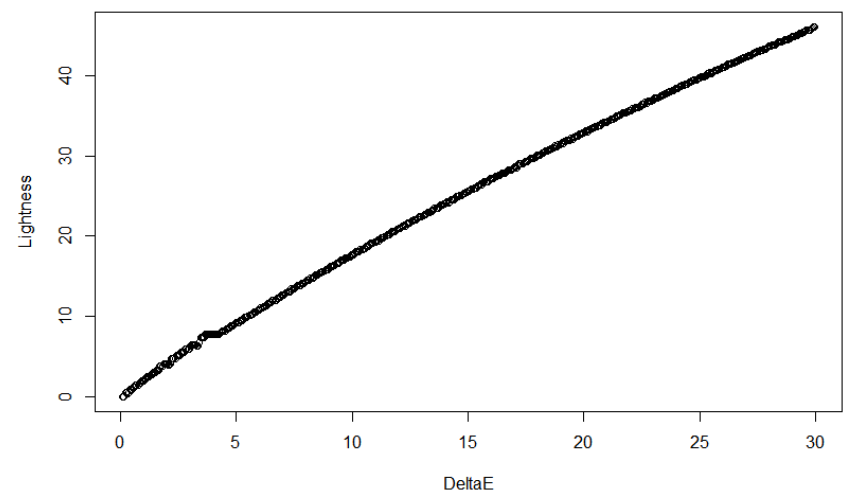

(c) $\Delta E_{00}$ vs. Lightness cut-offs

Fig. 5. Representation of the Module, Angle and Lightness cut-offs for each $\Delta E_{00}$ cut-off. 
Although the RMSE values are relatively small, regression errors still exist; hence, a $\Delta E_{00}$ difference between two colors lower than the $\Delta E_{00}$ cut-off may be considered higher than the derived decision boundary for either property, resulting in a error on a decision system that employed these models. To overcome this problem a small offset was added to the predicted values. This conservative approach assumes some loss of performance to advert a wrong decision. Fig. 6 illustrates the application of this offset in the predicted values of Lightness. The full line depicts the original predictions and the dotted line the shifted and utilized values.

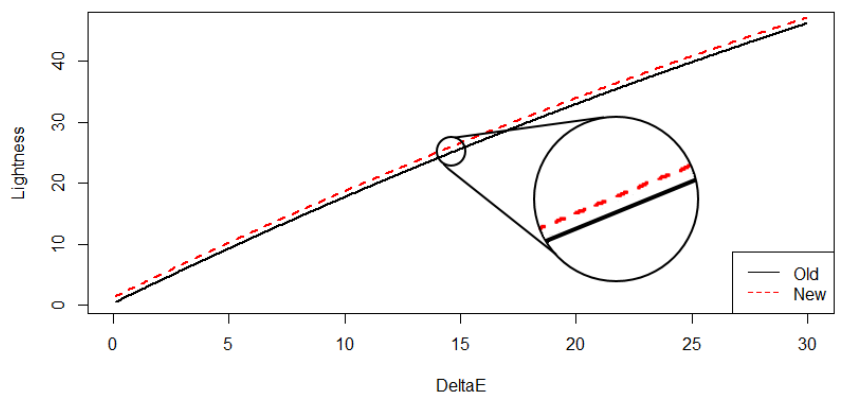

Fig. 6. Comparison between predicted and offset added values for the Lightness predictions.

In order to prove that our derived models were in fact precise on the obtained cut-offs for the properties, we generated an additional test set containing more than 150 million unique color comparisons, using different colors from the ones used in the training set. We then set multiple cut-off values for the $\Delta E_{00}$ and obtained the corresponding cut-offs for the properties using the models. Through the usage of these cutoffs, we verified on the test set if all of the pairs whose property values was above the corresponding cut-off, also had the $\Delta E_{00}$ value above the $\Delta E_{00}$ cut-off. We observed, as expected, that there were no errors in this evaluation, thus supporting the reliability of the models.

With these derived models it is then possible to automatically obtain, for any desired $\Delta E_{00}$ cut-off, decision boundaries for the Module, Angle and Lightness properties, so that any two colors whose difference surpasses those values can be classified as having a $\Delta E_{00}$ color difference higher than the specified cut-off. This is very important as it can be mapped into a structure that efficiently cuts calculations, resulting in a significant performance gain without jeopardizing $\Delta E_{00}$ benefits.

Each property and the corresponding threshold can be used to reduce the amount of $\Delta E_{00}$ calculations necessary for a given application that sets a $\Delta E_{00}$ cut-off. However, the color combinations that each property enables to cut are not the same. Hence, different strategies can be defined towards an efficient validation of whether its necessary to compute the full $\Delta E_{00}$ color difference or not.

\section{A. Cascade hypothesis}

One strategy for an efficient $\Delta E_{00}$-based color similarity decision consists on deploying the derived models into filtering modules on a cascade, as illustrated in Fig. 7. In each module of the cascade, simpler calculations are performed to compute Module, Angle or Lightness and an assessment is made. The full $\Delta E_{00}$ metric is only computed as last resource; hence, it will always be the last module of the cascade.

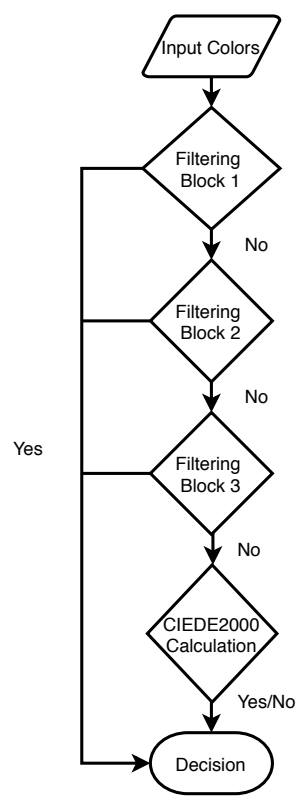

Fig. 7. Flowchart of the cascade strategy for deciding if a pair of colors is perceptually similar.

Each module of the cascade is intended to make a decision: colors are different and a cascade decision output is made; or, uncertainty exists and processing is passed to the next module of the cascade. Hence, it is expected that having faster modules (with smaller computation weight) executing first, preferably pruning as much comparisons as possible, will enable higher performance. Nevertheless, it is necessary to determine the most adequate cascade structure, i.e., to identify the optimal order of the operations. It is important to note that the cascade structure may be dependent on the hardware architecture. Moreover, the value of the $\Delta E_{00}$ cut-off to be considered may influence the best cascade structure due to the associated derived property cut-offs. Hence, for definition and validation of the cascade, several experiments were performed, as described in Section V-A. Additionally the performance of this cascade process can be improved by employing parallel strategies. This is illustrated in Fig. 8b, where an image is divided in several parts, and each one is processed separately.

\section{Evaluation Strategy and Results}

This section describes the assessment of the proposed methodologies, demonstrating their usability and performance gains, compared to direct applications of the $\Delta E_{00}$ formula. This assessment is divided into two subsections. The first subsection analysis the usability of the proposed properties in a cascade deployment using a simple practical application. For 
TABLE I

IDENTIFICATION OF POSSIBLE CASCADE STRUCTURES. EVEN THOUGH IT IS NOT REPRESENTED, THE $\Delta E_{00}$ BLOCK WILL ALWAYS BE THE LAST MODULE OF THE CASCADE.

\begin{tabular}{|c|c|c|c|c|}
\hline \multirow{2}{*}{$\#$} & \multicolumn{3}{|c|}{ Order in cascade } & \multirow{2}{*}{ ID } \\
\cline { 2 - 4 } & 1 & 2 & 3 & \\
\hline \hline 1 & Module & - & - & M \\
\hline 2 & Angle & - & - & A \\
\hline 3 & Lightness & - & - & L \\
\hline 4 & Module & Angle & - & MA \\
\hline 5 & Module & Lightness & - & ML \\
\hline 6 & Angle & Module & - & AM \\
\hline 7 & Angle & Lightness & - & AL \\
\hline 8 & Lightness & Angle & - & LA \\
\hline 9 & Lightness & Module & - & LM \\
\hline 10 & Module & Angle & Lightness & MAL \\
\hline 11 & Module & Lightness & Angle & MLA \\
\hline 12 & Angle & Lightness & Module & ALM \\
\hline 13 & Angle & Module & Lightness & AML \\
\hline 14 & Lightness & Module & Angle & LMA \\
\hline 15 & Lightness & Angle & Module & LAM \\
\hline
\end{tabular}

this, multiple cascade possibilities are studied in both single and multi-core (i.e., with parallelization), as well as in different hardware architectures. In the second subsection, the proposal is assessed over a real application to determine its effectiveness and precision.

\section{A. Cascade Assessment}

The order of the filtering blocks in the proposed cascade strategy will certainly influence the performance of the overall process for obtaining a color similarity decision, more specifically the time reduction that can be obtained with this strategy, compared to the direct calculation of the $\Delta E_{00}$ metric. Given the three properties put forward in this paper, there are 15 possible combinations for the cascade, as identified in Table I.

Due to the nature of the filtering modules, each of them will prune different color pairs, influencing the overall performance of the cascade. To evaluate all these possibilities, we implemented a simple segmentation algorithm that, given an image, a color model and a $\Delta E_{00}$ cut-off, produces a binary image that highlights only the pixels with a color difference to the model bellow the set cut-off. An illustration of the algorithm for both single core and multi-core (with division of the data) approaches is presented in Fig. 8. The idea behind the parallel approach is that, since similarity between each pixel in the image and the model is independent, the image can be divided into parts and process each part on different cores, maximizing the usage of the CPU.

In order to provide an unbiased evaluation on this algorithm, we selected as models the unique colors represented in the color bank [36]. We then extracted a representative subset of the images from the Caltech 256 [34] and the RAISE [37] datasets, which is composed of $4 \mathrm{~K}$ resolution images, and applied our algorithm on each image on this subset. Fig. 9 depicts samples of the images used in this experiment.

The performance evaluation of this algorithm was performed in three different computer architectures to understand the impact of the cascade structure in different hardware. Specifically, the following architectures were used: (1) a desktop computer equipped with an Intel i7 4471 processor @ 3.5Ghz; (2) a Raspberry Pi 2 Model B; (3) a Samsung Galaxy
TABLE II

CASCADE PERFORMANCE ON A DESKTOP COMPUTER FOR A $\Delta E_{00}=3$ FOR THE CALTECH 256 DATASET USING A SINGLE CORE APPROACH.

\begin{tabular}{|c|c|c|c|c|}
\hline \multicolumn{5}{|c|}{$\Delta E_{00}=3$} \\
\hline \hline $\begin{array}{c}\text { Cascade } \\
\text { Structure }\end{array}$ & $\begin{array}{c}\text { Time } \\
(\mathrm{sec})\end{array}$ & $\begin{array}{c}\text { CPU } \\
\text { Cycles }\end{array}$ & $\begin{array}{c}\text { Time } \\
\text { Reduction }\end{array}$ & $\begin{array}{c}\text { Cycles } \\
\text { Reduction }\end{array}$ \\
\hline \hline$\Delta E_{00}$ & 2121.82 & $7.427 \times 10^{12}$ & - & - \\
A & 712.254 & $2.492 \times 10^{12}$ & $-66.43 \%$ & $-66.45 \%$ \\
AL & 462.746 & $1.619 \times 10^{12}$ & $-78.19 \%$ & $-78.20 \%$ \\
AM & 87.064 & $3.047 \times 10^{11}$ & $-95.90 \%$ & $-95.90 \%$ \\
ALM & 98.578 & $3.450 \times 10^{11}$ & $-95.35 \%$ & $-95.35 \%$ \\
AML & 429.642 & $1.503 \times 10^{12}$ & $-79.75 \%$ & $-79.76 \%$ \\
L & 1319.91 & $4.617 \times 10^{12}$ & $-37.79 \%$ & $-37.83 \%$ \\
LA & 1166.08 & $4.080 \times 10^{12}$ & $-45.04 \%$ & $-45.07 \%$ \\
LM & 1129.93 & $3.953 \times 10^{12}$ & $-46.75 \%$ & $-46.77 \%$ \\
LAM & 1134.08 & $3.967 \times 10^{12}$ & $-46.55 \%$ & $-46.58 \%$ \\
LMA & 1129.67 & $3.952 \times 10^{12}$ & $-46.76 \%$ & $-46.78 \%$ \\
M & 45.911 & $1.607 \times 10^{11}$ & $\mathbf{- 9 7 . 8 4 \%}$ & $\mathbf{- 9 7 . 8 4 \%}$ \\
MA & 46.706 & $1.634 \times 10^{11}$ & $\mathbf{- 9 7 . 8 0 \%}$ & $\mathbf{- 9 7 . 8 0 \%}$ \\
ML & 57.502 & $2.014 \times 10^{11}$ & $\mathbf{- 9 7 . 2 9 \%}$ & $\mathbf{- 9 7 . 2 9 \%}$ \\
MAL & 58.111 & $2.034 \times 10^{11}$ & $\mathbf{- 9 7 . 2 6 \%}$ & $\mathbf{- 9 7 . 2 6 \%}$ \\
MLA & 58.094 & $2.034 \times 10^{11}$ & $\mathbf{- 9 7 . 2 6 \%}$ & $\mathbf{- 9 7 . 2 6 \%}$ \\
\hline
\end{tabular}

S6 smartphone. The algorithm was implemented in $\mathrm{C}++$ for the first two environments and in Java for the smartphone. In these experiments no explicit GPU acceleration was used and the CPU time and cycles allocated the process were measured. We chose to show the results obtained for four different $\Delta E_{00}$ cut-off values, namely $3,5,7,10$, because of their relation to the human notion of similarity [25]. For this evaluation, the cut-offs for the properties described by these $\Delta E_{00}$ values were obtained from the derived models, as described in Section III.

Table II details the results obtained on the desktop computer on all the proposed cascade strategies for a $\Delta E_{00}$ cut-off of 3 . In this table, two measures are compared, namely, the elapsed time for the processes and the amount of CPU cycles used. From these values its observable that the CPU cycles and the processing time required are directly proportional, as can also be observed in the percentage difference from the full formula calculation. In order to provide an additional comparison that illustrates the significancy of the temporal gains, we calculated the frames per second obtained using the $\Delta E_{00}$ approach and the MLA cascade and registered the values of 0.052 and 1.91, which clearly shows how much impact this methodology can have.

A summary of the time reductions obtained for the proposed cut-off values can be observed in Table III. These results show that, generally, filtering by Module as the first component of the cascade results in greater performance gains, which is consistent with the fact that the module is the less computationally demanding operation of the three proposed properties. This is particularly true as the $\Delta E_{00}$ cut-off value decreases; the lower the cut-off, the higher the amount of colors that can be discriminated by the properties. Using Lightness as the first block on the cascade leads to lower gains, as it is computationally more demanding than the other two properties. Moreover, these findings are consistent with the comment that, for the Module and Angle, the use of linear mapping functions are not the more efficient. It is also interesting to note that the largest gain is obtained with the combination Module-Angle; the addition of Lightness 


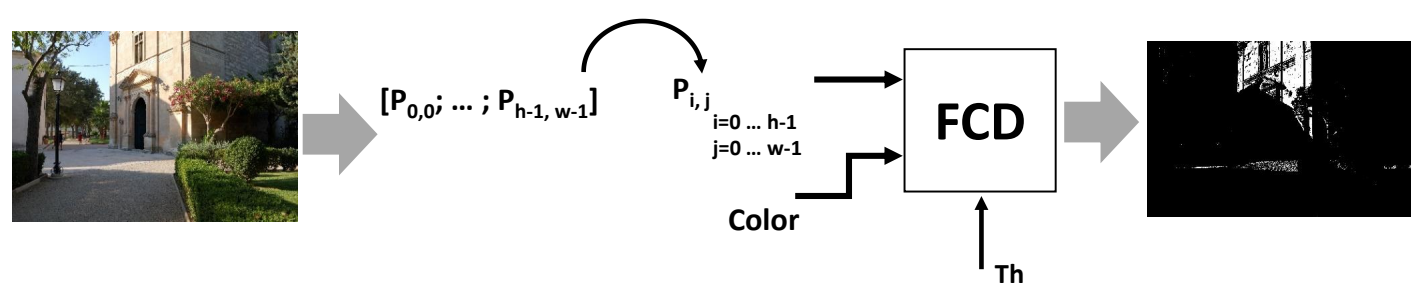

(a) Serial processing of the pixels in an image.

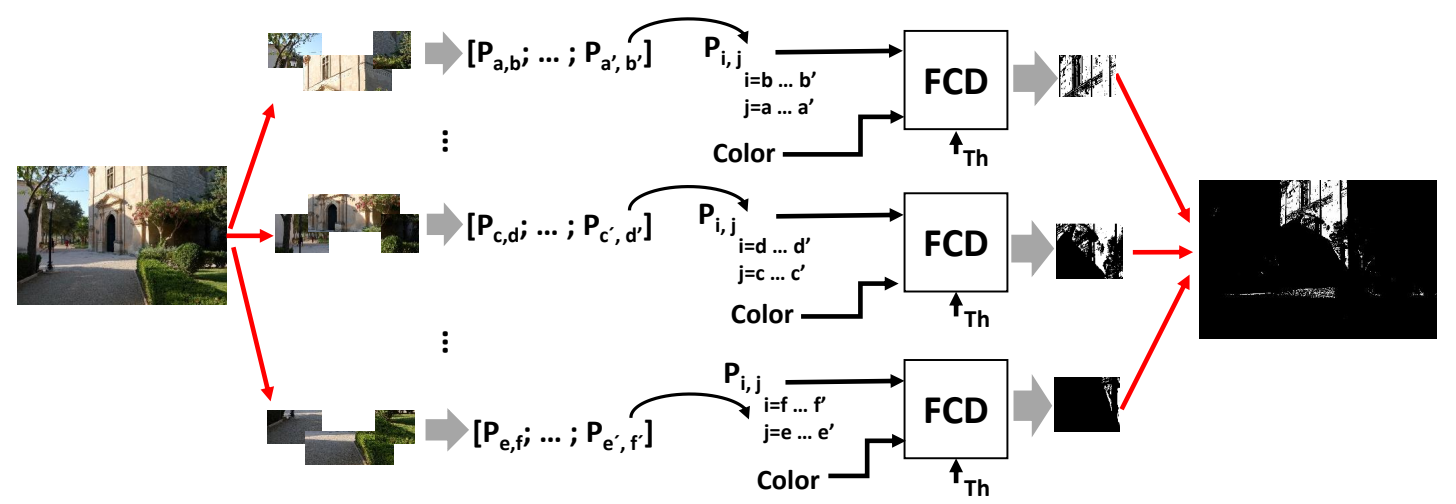

(b) Division of the data for parallel processing.

Fig. 8. Image processing possibilities taking advantage of CPUs with multiple cores. The Fast Color Decision (FCD) module represents one of the two proposed strategies.

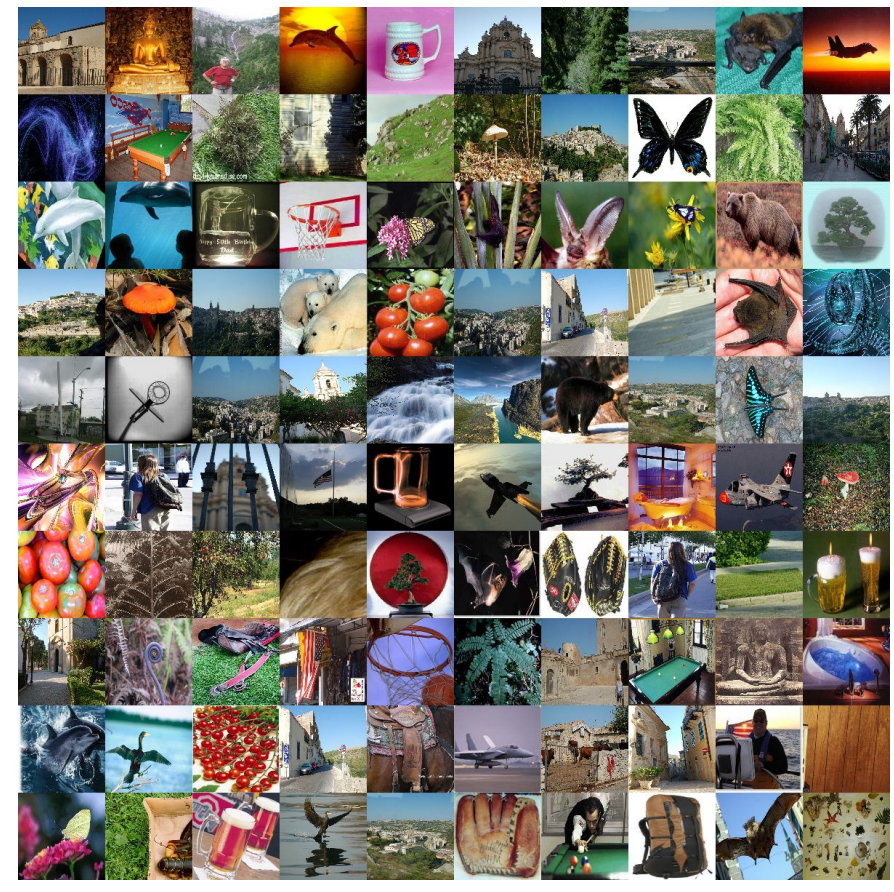

Fig. 9. Mosaic with samples from the three image datasets used in the study.

as the third block results in a slightly smaller gain. These observations are also true in the $4 \mathrm{~K}$ images of the dataset RAISE, as seen in Table IV. Due to the similarity between the results obtained in both datasets for all the cascades and
TABLE III

SUMMARY OF THE CASCADE RESULTS WHEN USING THE DESKTOP FOR THE CALTECH 256 DATASET USING A SINGLE CORE APPROACH.

\begin{tabular}{c|c|c|c|c|}
\cline { 2 - 5 } \multicolumn{1}{c|}{} & \multicolumn{4}{|c|}{ Relative Gains } \\
\hline \hline Cascade & $\Delta E_{00}=3$ & $\Delta E_{00}=5$ & $\Delta E_{00}=7$ & $\Delta E_{00}=10$ \\
\hline Struct. & $-66.43 \%$ & $-51.24 \%$ & $-50.75 \%$ & $-36.68 \%$ \\
A & $-78.19 \%$ & $-66.23 \%$ & $-63.48 \%$ & $-47.83 \%$ \\
AL & $-95.90 \%$ & $-85.73 \%$ & $-85.46 \%$ & $-\mathbf{- 7 1 . 3 3 \%}$ \\
ALM & $-95.35 \%$ & $-67.08 \%$ & $-64.88 \%$ & $-48.89 \%$ \\
AML & $-79.75 \%$ & $-85.44 \%$ & $-83.23 \%$ & $-67.21 \%$ \\
L & $-37.79 \%$ & $-32.46 \%$ & $-25.88 \%$ & $-17.98 \%$ \\
LA & $-45.04 \%$ & $-41.10 \%$ & $-38.36 \%$ & $-30.10 \%$ \\
LM & $-46.75 \%$ & $-41.21 \%$ & $-38.51 \%$ & $-27.16 \%$ \\
LAM & $-46.55 \%$ & $-42.49 \%$ & $-39.73 \%$ & $-31.15 \%$ \\
LMA & $-46.76 \%$ & $-42.48 \%$ & $-40.02 \%$ & $-31.36 \%$ \\
M & $\mathbf{- 9 7 . 8 4 \%}$ & $-85.03 \%$ & $-84.56 \%$ & $-66.01 \%$ \\
MA & $\mathbf{- 9 7 . 8 0 \%}$ & $\mathbf{- 8 7 . 4 6 \%}$ & $\mathbf{- 8 7 . 1 0 \%}$ & $-\mathbf{- 7 2 . 5 9 \%}$ \\
ML & $\mathbf{- 9 7 . 2 9 \%}$ & $-84.82 \%$ & $-81.95 \%$ & $-60.76 \%$ \\
MAL & $\mathbf{- 9 7 . 2 6 \%}$ & $\mathbf{- 8 7 . 1 3 \%}$ & $-84.87 \%$ & $-68.45 \%$ \\
MLA & $\mathbf{- 9 7 . 2 6 \%}$ & $-85.84 \%$ & $-83.42 \%$ & $-64.88 \%$ \\
\hline
\end{tabular}

hardware architectures, we chose to only show the tables with the results for the Caltech 256 dataset, as the extrapolated conclusions were the same.

The results of the same experiments performed on a Raspberry Pi 2 Model B are presented in Table V. Its noticeable that they are consistent with the results obtained on the desktop computer, with relative gains of the same order. We can also observe that the cascades starting with the Module are also the most efficient.

The rankings for the Android device are presented on Table VI, with overall relative gains lower than on the other 
TABLE IV

CASCADE PERFORMANCE ON A DESKTOP COMPUTER FOR A $\Delta E_{00}=5$ ON THE RAISE SUBSET USING A SINGLE CORE APPROACH. THE BEST RESULTS ARE HIGHLIGHTED.

\begin{tabular}{|c|c|c|}
\hline $\begin{array}{c}\text { Cascade } \\
\text { Structure }\end{array}$ & $\begin{array}{c}\text { Time } \\
(\mathrm{sec})\end{array}$ & $\begin{array}{c}\text { Time } \\
\text { Reduction }\end{array}$ \\
\hline$\Delta E_{00}$ & 13040.60 & - \\
$\mathrm{A}$ & 6641.31 & $-49.07 \%$ \\
$\mathrm{AL}$ & 4675.82 & $-64.14 \%$ \\
AM & 2020.31 & $-84.51 \%$ \\
ALM & 4530.91 & $-65.26 \%$ \\
AML & 2041.87 & $-84.34 \%$ \\
L & 8951.73 & $-31.35 \%$ \\
LA & 7802.66 & $-40.17 \%$ \\
LM & 7743.48 & $-40.62 \%$ \\
LAM & 7603.50 & $-41.69 \%$ \\
LMA & 7587.25 & $-41.82 \%$ \\
M & 2097.65 & $-83.91 \%$ \\
MA & 1802.53 & $\mathbf{- 8 6 . 1 8 \%}$ \\
ML & 2116.47 & $-83.77 \%$ \\
MAL & 1830.36 & $\mathbf{- 8 5 . 9 6 \%}$ \\
MLA & 1990.51 & $-84.74 \%$ \\
\hline
\end{tabular}

TABLE V

SUMMARY OF THE CASCADE RESULTS WHEN USING THE RASPBERRY P FOR THE CALTECH 256 DATASET USING A SINGLE CORE APPROACH.

\begin{tabular}{|c|c|c|c|c|}
\cline { 2 - 5 } \multicolumn{1}{c|}{} & \multicolumn{4}{|c|}{ Relative Gains } \\
\hline \hline Cascade & $\Delta E_{00}=3$ & $\Delta E_{00}=5$ & $\Delta E_{00}=7$ & $\Delta E_{00}=10$ \\
\hline \hline Structure & $-66.73 \%$ & $-51.35 \%$ & $-51.37 \%$ & $-36.78 \%$ \\
AL & $-80.30 \%$ & $-70.30 \%$ & $-67.08 \%$ & $-52.28 \%$ \\
AM & $-96.13 \%$ & $-86.36 \%$ & $-85.77 \%$ & $-\mathbf{7 1 . 5 8 \%}$ \\
ALM & $-82.09 \%$ & $-70.38 \%$ & $-68.68 \%$ & $-53.49 \%$ \\
AML & $-95.63 \%$ & $-86.48 \%$ & $-84.29 \%$ & $-69.13 \%$ \\
L & $-42.39 \%$ & $-38.93 \%$ & $-32.07 \%$ & $-23.66 \%$ \\
LA & $-50.78 \%$ & $-48.68 \%$ & $-45.13 \%$ & $-36.65 \%$ \\
LM & $\mathbf{- 5 2 . 7 0 \%}$ & $-48.77 \%$ & $-45.36 \%$ & $-33.70 \%$ \\
LAM & $\mathbf{- 5 2 . 4 7 \%}$ & $-48.95 \%$ & $-46.66 \%$ & $-37.83 \%$ \\
LMA & $\mathbf{- 5 2 . 6 1 \%}$ & $-48.18 \%$ & $-46.91 \%$ & $-37.98 \%$ \\
M & $\mathbf{- 9 7 . 9 5 \%}$ & $-85.18 \%$ & $-84.68 \%$ & $-66.07 \%$ \\
MA & $\mathbf{- 9 7 . 9 2} \%$ & $\mathbf{- 8 7 . 6 3 \%}$ & $\mathbf{- 8 7 . 2 8 \%}$ & $-\mathbf{7 2 . 7 1 \%}$ \\
ML & $\mathbf{- 9 7 . 4 4 \%}$ & $-86.00 \%$ & $-82.89 \%$ & $-62.67 \%$ \\
MAL & $\mathbf{- 9 7 . 4 3 \%}$ & $\mathbf{- 8 8 . 1 3 \%}$ & $-85.70 \%$ & $-70.03 \%$ \\
MLA & $\mathbf{- 9 7 . 4 3 \%}$ & $-87.04 \%$ & $-84.64 \%$ & $-67.37 \%$ \\
\hline
\end{tabular}

cases. Another interesting fact is that some cascades with the Angle as first block have higher performance gains than cascades starting with Module. Nevertheless, the gains are still very significant, ranging from $64 \%$ to $88 \%$. These differences in the results are undoubtedly related to the different CPU architectures and programming languages used. Nonetheless the results show that the gain of the proposed strategy is always significant.

Table VII details the summary of the relative gains obtained using the desktop computer using a parallel strategy. To perform this evaluation, we divided each input image into 4 equal parts, and issued a thread that processed the algorithm using each of these parts. Although the performance gains are similar to the results of the sequential approach presented in Table III, a slight loss in performance is noticed mainly due the thread overhead. This behaviour is analogous in all of the tested architectures and different $\Delta E_{00}$ cut-offs.

\section{B. Assessment in a real application}

In order to asses our proposal in a real application, we implemented a soccer player detection and team discrimination algorithm. To evaluate this application we used the dataset ISSIA [38], which consists of several videos of a game between
TABLE VI

SUMMARY OF THE CASCADE RESULTS WHEN USING THE SMARTPHONE FOR THE CALTECH 256 DATASET USING A SINGLE CORE APPROACH.

\begin{tabular}{|c|c|c|c|c|}
\cline { 2 - 5 } \multicolumn{1}{c|}{} & \multicolumn{4}{|c|}{ Relative Gains } \\
\hline \hline $\begin{array}{c}\text { Cascade } \\
\text { Structure }\end{array}$ & $\Delta E_{00}=3$ & $\Delta E_{00}=5$ & $\Delta E_{00}=7$ & $\Delta E_{00}=10$ \\
\hline \hline A & $-64.24 \%$ & $-48.75 \%$ & $-45.91 \%$ & $-32.32 \%$ \\
AL & $-77.92 \%$ & $-67.45 \%$ & $-64.05 \%$ & $-48.64 \%$ \\
AM & $-86.70 \%$ & $-78.89 \%$ & $-\mathbf{7 7 . 7 5 \%}$ & $-64.05 \%$ \\
ALM & $-78.71 \%$ & $-67.29 \%$ & $-63.15 \%$ & $-52.35 \%$ \\
AML & $\mathbf{- 8 8 . 1 3 \%}$ & $\mathbf{- 7 9 . 2 3 \%}$ & $-75.30 \%$ & $\mathbf{- 6 4 . 8 7 \%}$ \\
L & $-46.48 \%$ & $-37.00 \%$ & $-27.62 \%$ & $-23.78 \%$ \\
LA & $-53.94 \%$ & $-48.62 \%$ & $-43.96 \%$ & $-35.03 \%$ \\
LM & $-52.03 \%$ & $-47.14 \%$ & $-43.64 \%$ & $-33.02 \%$ \\
LAM & $-53.73 \%$ & $-45.92 \%$ & $-43.41 \%$ & $-37.93 \%$ \\
LMA & $-53.00 \%$ & $-47.42 \%$ & $-40.44 \%$ & $-38.90 \%$ \\
M & $-88.14 \%$ & $-75.42 \%$ & $-74.30 \%$ & $-55.87 \%$ \\
MA & $\mathbf{- 8 8 . 5 0 \%}$ & $\mathbf{- 7 8 . 2 1 \%}$ & $-\mathbf{7 7 . 7 5 \%}$ & $\mathbf{- 6 5 . 2 5 \%}$ \\
ML & $-86.72 \%$ & $-76.81 \%$ & $-74.26 \%$ & $-57.36 \%$ \\
MAL & $-86.96 \%$ & $\mathbf{- 7 8 . 8 5 \%}$ & $-76.23 \%$ & $-63.65 \%$ \\
MLA & $-86.91 \%$ & $-77.90 \%$ & $-74.84 \%$ & $-61.53 \%$ \\
\hline
\end{tabular}

TABLE VII

SUMMARY OF THE DATA DIVISION RESULTS WHEN USING THE DESKTOP FOR THE CALTECH 256 DATASET.

\begin{tabular}{|c|c|c|c|c|}
\hline & \multicolumn{4}{|c|}{ Relative Gains } \\
\hline $\begin{array}{c}\text { Cascade } \\
\text { Struct. }\end{array}$ & $\Delta E_{00}=3$ & $\Delta E_{00}=5$ & $\Delta E_{00}=7$ & $\Delta E_{00}=10$ \\
\hline $\mathrm{A}$ & $-63.41 \%$ & $-45.87 \%$ & $-48.35 \%$ & $-38.68 \%$ \\
\hline $\mathrm{AL}$ & $-75.86 \%$ & $-63.88 \%$ & $-60.21 \%$ & $-46.93 \%$ \\
\hline $\mathrm{AM}$ & $-95.08 \%$ & $-82.20 \%$ & $-81.81 \%$ & $-67.82 \%$ \\
\hline ALM & $-77.88 \%$ & $-64.89 \%$ & $-58.57 \%$ & $-49.33 \%$ \\
\hline AML & $-94.38 \%$ & $-81.73 \%$ & $-76.92 \%$ & $-63.49 \%$ \\
\hline $\mathrm{L}$ & $-35.70 \%$ & $-31.64 \%$ & $-23.98 \%$ & $-17.98 \%$ \\
\hline LA & $-43.41 \%$ & $-40.29 \%$ & $-36.86 \%$ & $-33.36 \%$ \\
\hline LM & $-46.86 \%$ & $-40.99 \%$ & $-37.89 \%$ & $-28.50 \%$ \\
\hline LAM & $-45.63 \%$ & $-42.09 \%$ & $-33.74 \%$ & $-34.20 \%$ \\
\hline LMA & $-45.98 \%$ & $-42.19 \%$ & $-34.00 \%$ & $-34.26 \%$ \\
\hline M & $-97.19 \%$ & $-81.65 \%$ & $-81.28 \%$ & $-62.90 \%$ \\
\hline MA & $-97.15 \%$ & $-\mathbf{8 3 . 8 8 \%}$ & $-\mathbf{8 3 . 3 8 \%}$ & $-68.89 \%$ \\
\hline ML & $-96.43 \%$ & $-81.58 \%$ & $-77.72 \%$ & $-59.07 \%$ \\
\hline MAL & $-96.37 \%$ & $-83.46 \%$ & $-78.65 \%$ & $-65.21 \%$ \\
\hline MLA & $-96.37 \%$ & $-82.41 \%$ & $-77.30 \%$ & $-62.32 \%$ \\
\hline
\end{tabular}

two teams. This dataset also provides manual annotations of the positions of each player in the full length of the streams, and has been used in several research papers [39], [40]. We choose to use this dataset to test validate our hypothesis in a real world application, while also enabling objective evaluation and reproducibility. Fig. 10 illustrates a sample frame from two cameras used in the dataset.

The developed algorithm uses team color information to create a binary segmentation mask for each team, and to detect blobs that are player candidates. The blobs that are too big or too small are filtered and the resulting blobs are used to define the corresponding bounding boxes. To obtain the segmentation masks, we used the same approach as defined in subsection $\mathrm{V}-\mathrm{A}$, and used a $\Delta E_{00}$ cut-off of 7 in order to account for the differences in illumination in the streams. Fig. 11 shows the result of applying the steps 1 through 4 for the frame 50 of the stream 4

This real application was used for a two-fold assessment of our proposal. First, we evaluated if the segmentation masks obtained for each team using just the $\Delta E_{00}$ formula was equal to the segmentation masks obtained by application of the cascade methodology. A second evaluation was made by comparing the results of the implemented algorithm with the 


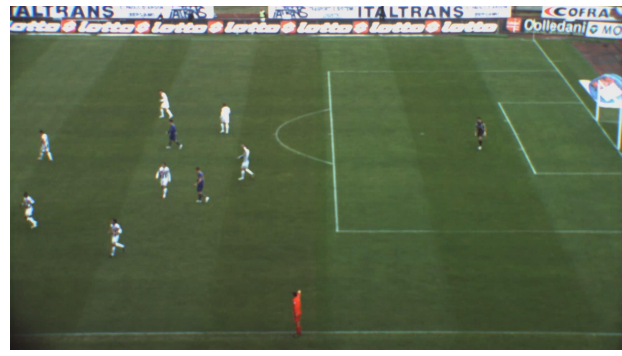

(a) Frame 35 from the stream 1.

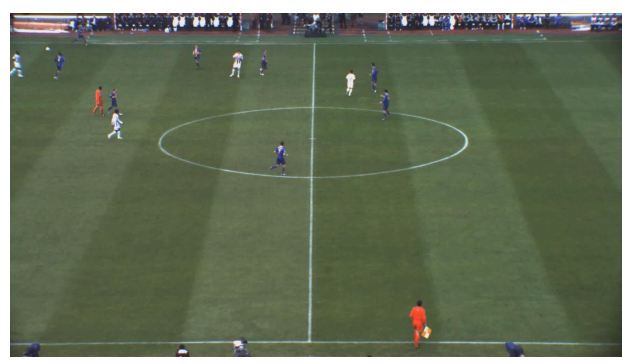

(b) Frame 50 from the stream 4.

Fig. 10. Sample frames for two different cameras of the ISSIA dataset.

dataset's ground truth, while measuring the processing times. For this assessment, we show the results for the two most notable cascade strategies, namely the MAL and MA, since the observed behaviour is the same as on the previous application.

The segmentation masks, for each team, obtained through the direct used of $\Delta E_{00}$ and with the proposed cascade approach, were compared to assess the coherence of the results and validate the cascade outcome. The comparison of the masks, for each frame of the dataset videos, was performed through the calculation of the Hamming distance. The result was a zero distance, demonstrating that the proposed cascade produced exactly the same mask.

The second evaluation was made by comparing the results of the implemented algorithm with the dataset ground truth, while measuring the processing time. These results are summarised in Table VIII, for the different $\Delta E_{00}$ cut off values and cascades. Even though the implemented algorithm was somewhat straightforward and not optimised, we obtained a mean precision and recall of $92.5 \%$ and $75.8 \%$ respectively. The processing times of Table VIII support the previous observations, showing significant gains by using the proposed cascade strategy.

TABLE VIII

AVERAGE PROCESSING TIME AND FPS OBTAINED FOR THE STREAMS OF THE DATASET ISSIA.

\begin{tabular}{|l|l|l|l|l|}
\hline $\begin{array}{c}\text { Cascade } \\
\text { Struct. }\end{array}$ & Time(s) & FPS & Time(s) & FPS \\
\hline \hline$\Delta E_{00}$ & 1511.42 & 1.99 & 1542.01 & 1.95 \\
MAL & 240.17 & 13.05 & 664.92 & 4.64 \\
MA & 238.69 & 12.27 & 867.27 & 3.46 \\
\hline
\end{tabular}

\section{CONCLUSIONS}

The CIEDE2000 metric targets an important issue in computer vision - the perceptual difference in colors - with direct

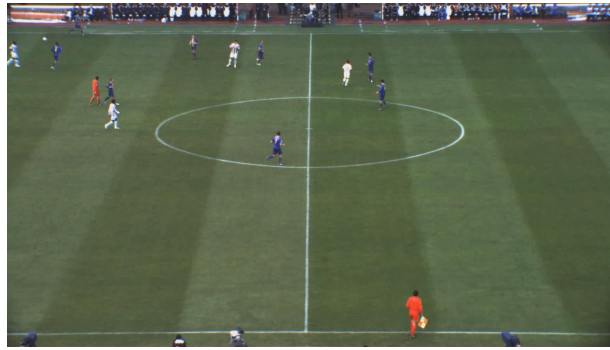

(a) Original frame 50 from the stream 4 .

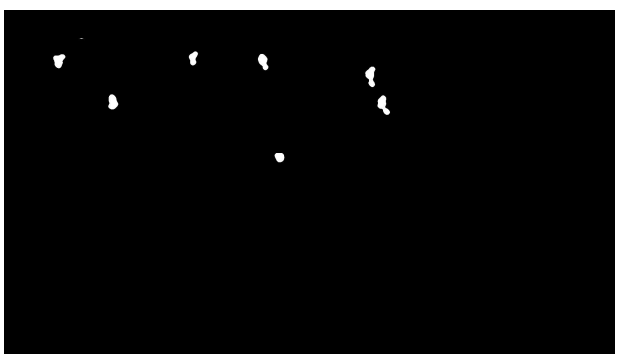

(b) Blobs detected bellonging to the blue team.

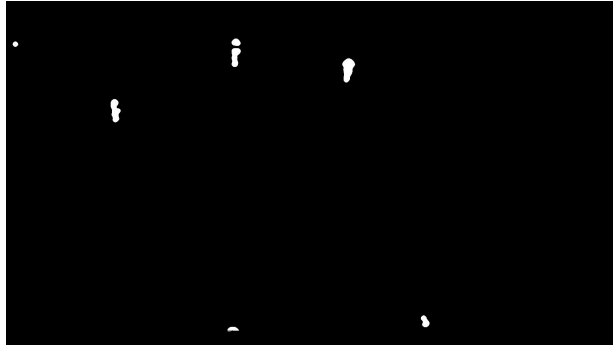

(c) Blobs detected bellonging to the white team.

Fig. 11. Detected blobs from the frame 50 of the stream 4 .

impact and application to image and video processing. This formulation has several recognized advantages with regards to other state-of-the-art strategies, a fact supported by the classification of CIEDE2000 metric as an international standard and its usage in many industry applications. Despite the recognized advantages and the fact that it provides results consistent to the visual perception $(\Delta V)$, its adoption has been impaired by the high mathematical complexity and computational cost; a drawback that becomes more relevant when processing large amounts of data such as images of increasing resolution or video streams.

This paper proposes a new paradigm for the application of the CIEDE2000 metric to computer vision, with strategies that allow a faster decision about color similarity based upon simple properties and derived models. The contributions of this paper can be summarized in four different steps. In the first step, we analyse the CIEDE2000 formula and identify an initial set of properties to be used for decision and filtering color pairs. An underlying hypothesis was formulated: if a given CIEDE2000 color similarity cut-off is set, then it is possible to obtain at least one mapping function that produces a corresponding cut-off on any property complying with the CIEDE2000 metric. Secondly, the usability and validity of the proposed properties was mathematically demonstrated, and new mapping models were defined through the usage of 
regression techniques, trained on experimental data. Finally, the proposed models were combined into a cascade approach, in order to take advantage of the filtering modules and the identified relations for a significantly faster computation and decision of color differences.

The proposed filtering properties were: the euclidean distance between two RGB vectors, the angle between two RGB vectors and the absolute difference between the L components of two colors in the CIELAB color space, regarded as Module, Angle and Lightness, respectively. This enable very high processing gains using different strategies and hardware platforms. Nevertheless, other properties can be analysed and their benefits studied. From the evaluations performed using these properties, it was observed that the order of the filtering modules in a cascade strategy had a significant impact in the resulting processing time. This suggests that a vectorized approach using these properties would result in overall higher processing times, as the calculation of the features would be required at the start of the processing chain.

Even though the hardware platforms and programming languages had a slight impact on the results, the proposed strategies enabled very significant gains in all the experiments. These showed a dependence on the desired decision boundary on the result of $\Delta E_{00}$, but were always very high for useful values of the metric. In the experiments, $\Delta E_{00}$ cut-off values above 10 were not considered as such distance is deemed to correspond to notably different colors. Nevertheless, the knowledge resulting from the reported experiments and proposed models enables implementations in a way that it can adapt itself to different decision thresholds and hardware platforms known a priori and given a context passed as input. Additionally, derivation of the models can be optimized by taking into consideration application centred color sub-spaces. Moreover, additional properties can be studied and included. In this case, a sensitivity analysis can be of great value to avoid testing each cascade possibility.

The best structure for the cascade strategy presented slight variations, but overall a small set of possibilities can be identified, to be used horizontally with very small decreases in the performance gain. When considering the data division approach, the results are in line with what was expected and a preferred cascade strategy that can be generically applied can also be identified and, as expected, the overall processing time is smaller with data division, as long as there are multi-core CPUs available.

\section{ACKNOWLEDGEMENTS}

The work presented was partially supported by the following projects: "TEC4Growth - Pervasive Intelligence, Enhancers and Proofs of Concept with Industrial Impact/NORTE01-0145-FEDER-000020" is financed by the North Portugal Regional Operational Programme (NORTE 2020), under the PORTUGAL 2020 Partnership Agreement, and through the European Regional Development Fund (ERDF); MOG CLOUD SETUP - No17561, supported by Norte Portugal Regional Operational Programme (NORTE 2020), under the PORTUGAL 2020 Partnership Agreement, through the Eu- ropean Regional Development Fund (ERDF) and project FotoInMotion funded by H2020 Framework Programme of the European Commission.

\section{REFERENCES}

[1] T. R. Mengko, Y. Susilowati, R. Mengko, and B. E. Leksono, "Digital image processing technique in rock forming minerals identification," in Circuits and Systems, 2000. IEEE APCCAS 2000. The 2000 IEEE AsiaPacific Conference on. IEEE, 2000, pp. 441-444.

[2] R. Ghinea, M. M. Pérez, L. J. Herrera, M. J. Rivas, A. Yebra, and R. D. Paravina, "Color difference thresholds in dental ceramics," Journal of Dentistry, vol. 38, pp. e57-e64, 2010.

[3] G. L. Main and J. R. Morris, "Effect of macerating enzymes and postfermentation grape-seed tannin on the color of cynthiana wines," American journal of enology and viticulture, vol. 58, no. 3, pp. 365372, 2007.

[4] L. P. Tong, B. Peng, Y. W. Fei, and H. W. Yang, "Application of ciede 2000 color difference formula in jet fuel silver strip corrosion color recognition," in Key Engineering Materials, vol. 531. Trans Tech Publ, 2013, pp. 54-57.

[5] M. R. Luo, G. Cui, and B. Rigg, "The development of the cie 2000 colour-difference formula: Ciede2000," Color Research \& Application, vol. 26, no. 5, pp. 340-350, 2001.

[6] M. Melgosa, L. Gómez-Robledo, P. García, S. Morillas, C. FernándezMaloigne, N. Richard, M. Huang, C. Li, and G. Cui, "Color-quality control using color-difference formulas: progress and problems," in Third International Conference on Applications of Optics and Photonics, vol. 10453. International Society for Optics and Photonics, 2017, p. 104530U.

[7] S. Aligholi, R. Khajavi, and M. Razmara, "Automated mineral identification algorithm using optical properties of crystals," Computers \& Geosciences, vol. 85, pp. 175-183, 2015.

[8] G. Sharma, W. Wu, and E. N. Dalal, "The ciede2000 color-difference formula: Implementation notes, supplementary test data, and mathematical observations," Color Research \& Application, vol. 30, no. 1, pp. 21-30, 2005.

[9] G. Sharma, "The ciede2000 color-difference formula," http://www2.ece. rochester.edu/ $\sim$ gsharma/ciede2000/, accessed March 7, 2018).

[10] A. R. Robertson, "Historical development of cie recommended color difference equations," Color Research \& Application, vol. 15, no. 3, pp. 167-170, 1990.

[11] _ " "The cie 1976 color-difference formulae," Color Research \& Application, vol. 2, no. 1, pp. 7-11, 1977.

[12] R. McDonald and K. J. Smith, "Cie94-a new colour-difference formula," Journal of the Society of Dyers and Colourists, vol. 111, no. 12, pp. 376-379, 1995.

[13] B. J. Lindbloom, "Delta e (cmc)," Brucelindbloom. com. Revised, pp. 04-16, 2009.

[14] K. Richter, S. Brako, G. Cui, M. R. Luo, M. Melgosa, and T. Seim, "Validity of formulae for predicting small colour differences," CIE, International Commision on Illumination, Tech. Rep., 2019, CIE 230:2019.

[15] J. C. Ragain and W. M. Johnston, "Color acceptance of direct dental restorative materials by human observers," Color Research \& Application, vol. 25, no. 4, pp. 278-285, 2000.

[16] A. G. Wee, D. T. Lindsey, K. M. Shroyer, and W. M. Johnston, "Use of a porcelain color discrimination test to evaluate color difference formulas," The Journal of prosthetic dentistry, vol. 98, no. 2, pp. 101-109, 2007.

[17] R. Seghi, W. Johnston, and W. O'Brien, "Performance assessment of colorimetric devices on dental porcelains," Journal of Dental Research, vol. 68, no. 12, pp. 1755-1759, 1989.

[18] A. G. Wee, D. T. Lindsey, S. Kuo, and W. M. Johnston, "Color accuracy of commercial digital cameras for use in dentistry," Dental Materials, vol. 22, no. 6, pp. 553-559, 2006.

[19] W. J. O'Brien, K. M. Boenke, and C. L. Groh, "Coverage errors of two shade guides." International Journal of Prosthodontics, vol. 4, no. 1, 1991.

[20] F. Bayindir, S. Kuo, W. M. Johnston, and A. G. Wee, "Coverage error of three conceptually different shade guide systems to vital unrestored dentition," The journal of prosthetic dentistry, vol. 98, no. 3, pp. 175185, 2007.

[21] O. E. Pecho, R. Ghinea, R. Alessandretti, M. M. Pérez, and A. Della Bona, "Visual and instrumental shade matching using cielab and ciede2000 color difference formulas," Dental Materials, vol. 32, no. 1, pp. 82-92, 2016. 
[22] H. Liu, M. Huang, G. Cui, M. R. Luo, and M. Melgosa, "Colordifference evaluation for digital images using a categorical judgment method," JOSA A, vol. 30, no. 4, pp. 616-626, 2013.

[23] C. Gómez-Polo, M. P. Muñoz, M. C. L. Luengo, P. Vicente, P. Galindo, and A. M. M. Casado, "Comparison of the cielab and ciede2000 color difference formulas," The Journal of prosthetic dentistry, vol. 115, no. 1, pp. 65-70, 2016.

[24] Y. Yang, J. Ming, and N. Yu, "A design method of saturation test image based on ciede2000," Advances in Multimedia, vol. 2012, p. 14, 2012.

[25] — "Color image quality assessment based on ciede2000," Advances in Multimedia, vol. 2012, p. 11, 2012.

[26] S. Liu, M. S. Brown, S. J. Kim, and Y.-W. Tai, "Colorization for single image super resolution," in European Conference on Computer Vision. Springer, 2010, pp. 323-336.

[27] I. Lissner and P. Urban, "How perceptually uniform can a hue linear color space be?" in Color and Imaging Conference, vol. 2010, no. 1. Society for Imaging Science and Technology, 2010, pp. 97-102.

[28] S. Schwarz, M. Sjöström, and R. Olsson, "Improved edge detection for ewoc depth upscaling," in 2012 19th International Conference on Systems, Signals and Image Processing (IWSSIP). IEEE, 2012, pp. $1-4$.

[29] C. Stauffer and W. E. L. Grimson, "Adaptive background mixture models for real-time tracking," in Computer Vision and Pattern Recognition, 1999. IEEE Computer Society Conference on., vol. 2. IEEE, 1999, pp. 246-252.

[30] K. He, G. Gkioxari, P. Dollár, and R. Girshick, "Mask r-cnn," in Computer Vision (ICCV), 2017 IEEE International Conference on. IEEE, 2017, pp. 2980-2988.

[31] R. Dony and S. Wesolkowski, "Edge detection on color images using rgb vector angles," in Electrical and Computer Engineering, 1999 IEEE Canadian Conference on, vol. 2. IEEE, 1999, pp. 687-692.

[32] S. Wesolkowski, R. D. Dony, and M. Jernigan, "Global color image segmentation strategies: Euclidean distance vs. vector angle," in Neural Networks for Signal Processing IX, 1999. Proceedings of the 1999 IEEE Signal Processing Society Workshop. IEEE, 1999, pp. 419-428.

[33] H. R. Kang, Computational color technology. Spie Press Bellingham, 2006.

[34] G. Griffin, A. Holub, and P. Perona, "Caltech-256 object category dataset," 2007.

[35] A. Liaw and M. Wiener, "Classification and regression by randomforest," $R$ news, vol. 2, no. 3, pp. 18-22, 2002.

[36] "Color names supported by all browsers," http://www.w3schools.com/ colors/colors_names.asp, [Online; accessed 03-01-2019].

[37] D.-T. Dang-Nguyen, C. Pasquini, V. Conotter, and G. Boato, "Raise: a raw images dataset for digital image forensics," in Proceedings of the 6th ACM Multimedia Systems Conference. ACM, 2015, pp. 219-224.

[38] T. D'Orazio, M. Leo, N. Mosca, P. Spagnolo, and P. L. Mazzeo, "A semiautomatic system for ground truth generation of soccer video sequences," in Advanced Video and Signal Based Surveillance, 2009. AVSS'09. Sixth IEEE International Conference on. IEEE, 2009, pp. 559-564.

[39] H. B. Shitrit, J. Berclaz, F. Fleuret, and P. Fua, "Tracking multiple people under global appearance constraints," 2011.

[40] _ - "Multi-commodity network flow for tracking multiple people," IEEE transactions on pattern analysis and machine intelligence, vol. 36 no. 8, pp. 1614-1627, 2014.

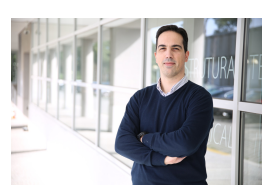

Pedro Carvalho received the degree in electrical and computer engineering in 2001, the M.Sc. degree network and communication services in 2004 and the $\mathrm{Ph} . \mathrm{D}$ degree in electrical and computers engineering in 2012, from the Faculty of Engineering, University of Porto, Portugal. He joined INESC TEC in 2001 and is currently a senior researcher at the Center of Telecommunications and Multimedia. He has been an invited adjunct professor at the School of Engineering of the Polytechnic Institute of Porto, Porto, Portugal since 2014. His research interests include image/video processing and computer vision.

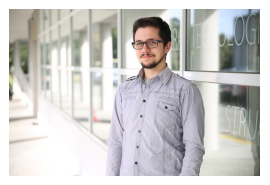

Gil Coelho received the degree and M.Sc. degree in Electrical and Computer Engineering in 2014, from the Faculty of Engineering, University of Porto, Portugal. He joined INESC TEC in 2014 and is currently a researcher at the Center of Telecommunications and Multimedia. He was working on projects on detecting and tracing people in the retail environment. His research interests include image/video processing, image/video enhancement and computer vision.

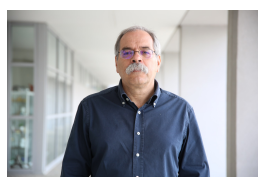

Luís Côrte-Real was born in Vila do Conde, Portugal, in 1958. He received the degree in Electrical Engineering from the Faculty of Engineering, University of Porto, Portugal, in 1981, the M.Sc. degree in Electrical and Computers Engineering in 1986 from Instituto Superior Técnico, Universidade Técnica de Lisboa, Lisbon, Portugal and the Ph.D degree from the Faculty of Engineering, University of Porto, in 1994. In 1984 he joined University of Porto as a lecturer of telecommunications. He is currently an associate professor at the Department of Electrical and Computer Engineering in the Faculty of Engineering, University of Porto. He is a researcher at INESC TEC since 1985. His research interests include image/video processing and coding.

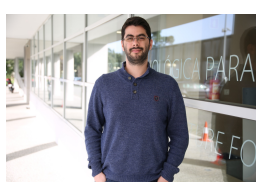

Américo Pereira received the degree and M.Sc. in computer science in 2011 and 2013 from the Faculty of Science, University of Porto, Portugal. He jointed INESC TEC in 2014 and is a researcher in the Center of Telecommunications and Multimedia. $\mathrm{He}$ is also a Ph.D student since 2015 in electrical and computer engineering on the Faculty of Engineering, University of Porto, Portugal. His research goals are related to computer vision and image/video processing, with an emphasis on machine learning. 